\title{
Marketed under Investigational New Drug
}

National Cancer Institute

\section{Source}

National Cancer Institute. Marketed under Investigational New Drug. NCI Thesaurus. Code C75302.

A category specifying that a product is marketed under an Investigational New Drug Application. 